\title{
Sistemas productivos agroecológicos
}

\author{
Agroecological production systems \\ Roa Vega María Ligia ${ }^{1}$ \\ 'Zootecnista, MSc. Docente Universidad de los Llanos \\ mroa@unillanos.edu.co
}

Recibido 04 de Noviembre 2015, Aceptado 29 de Abril 2016

\section{RESUMEN}

La agroecología es una disciplina que provee las bases científicas y metodológicas para estudiar, manejar y evaluar agroecosistemas de una manera holística, la cual ha emergido como una opción tecnológica válida para el manejo de los recursos naturales puesto que, entre otras cosas, incorpora acciones sociales colectivas de carácter participativo, permitiendo el diseño de sistemas agropecuarios sostenibles como pilar de desarrollo que apunta al fundamento de la crisis ecológica y social de la producción campesina e industrial-capitalista en la región. En ese sentido, la agroecología no solo se centra en los cultivos y animales sino en la sostenibilidad ecológica, socioeconómica y cultural del sistema de producción. Además de suministrar una base científica para alcanzar una productividad sostenible, la agroecología enfatiza la capacidad de las comunidades locales para innovar, evaluar, y adaptarse a condiciones heterogéneas extremas, a través de métodos de investigación participativa y de extensión campesino a campesino. Las tecnologías agroecológicas enfatizan en la diversidad, la sinergia, el reciclaje e integración, y en los procesos sociales para que valoren la participación de la comunidad, que es el recurso humano clave, como piedra angular de cualquier estrategia que apunte a aumentar las opciones de la participación de población rural, sobre todo productores de escasos recursos. El enfoque de campesino a campesino es justamente, un aspecto de relevancia a tener en cuenta en la propuesta del pensamiento agroecológico, atendiendo por supuesto a la inclusión de profesionales técnicos como facilitadores del proceso, quienes en principio deben comprender la complejidad 
del sistema agropecuario y sus interrelaciones antropológicas, sociales, económicas, políticas y ecosistémicas, para apoyar efectivamente los procesos de generación y transferencia de conocimientos. En conclusión, los diseños agroecológicos son propios en contexto local y tiene sus particularidades desde cada dimensión de la sostenibilidad entre los cuales se consideran aspectos: Culturales, ecológicos, políticos, productivos y económicos, entre otros; de esta manera, se define que los diseños agroecológicos se construyen a partir del análisis de contexto y de las necesidades puntuales existentes por los productores (sociocultural) quienes son los responsables y dolientes directos de los procesos agropecuarios en cada región.

Palabras clave: Agrosistemas, investigación participativa, procesos agroecológicos.

\section{ABSTRACT}

The agroecology is a discipline that provides the scientific and methodological basis for study, manage and evaluate agro-ecosystems in a holistic way, which it has emerged as a valid technological option for the management of natural resources since, among other things, incorporates collective social actions of a participatory nature, enabling the design of sustainable farming systems as a pillar of development that points to the foundation of ecological and social crisis of peasant and industrial-capitalist production in the region. In that sense, the agroecology not only focuses on crops and animals but on ecological sustainability, socioeconomic and cultural of production system. Addition to supplying a scientific basis for achieving sustainable productivity, the agroecology emphasizes the ability of local communities to innovate, evaluate, and adapt to extreme heterogeneous conditions, through participatory research methods and farmer-to-farmer extension. The agroecological technologies emphasize in the diversity, synergy, recycling and integration, and in the social processes that value community involvement, which is the key human resource, as the cornerstone of any strategy aimed at increasing the options involving rural population, especially farmers with scarce resources. The approach farmer to farmer is precisely, one aspect of relevance to consider in 
the proposal of agroecological thought, attending of course to the inclusion of technical professionals as facilitators of the process, who in principle should understand the complexity of the agricultural system and its anthropological, social, economic, political and ecosystems relationships, In conclusion, the agroecological designs are unique in the local context and has its peculiarities from each dimension of sustainability including aspects considered as: Cultural, ecological, political, productive and economic, among others; in this way, it define that agroecological designs are built from the analysis of context and existing specific needs by producers (sociocultural) who are responsible and direct mourners of agricultural processes in each region.

Keywords: Agrosystems, participatory research, agro-ecological processes.

\section{RESUMO}

O agroecologia é uma disciplina que fornece a base científica e metodológica para o estudo, gerir e avaliar os agro-ecossistemas de uma forma holística, que emergiu como um opção tecnológica válido para a gestão dos recursos naturais, já que, entre outras coisas, incorpora a ação social coletiva de participação, permitindo o desenho de sistemas agrícolas sustentáveis como um pilar do desenvolvimento que aponta a fundação da crise ecológica e social dos camponeses ea produção industrial-capitalista na região. Nesse sentido, o agroecologia não só incide sobre as culturas e animais, mas sobre a sustentabilidade ecológica, socioeconômico e cultural o sistema de produção. Além de fornecer uma base científica para alcançar uma produtividade sustentável, o agroecologia enfatiza a capacidade das comunidades locais para inovar, avaliar e adaptar-se às condições heterogêneas extremas, através de métodos de pesquisa participativa e extensão de agricultor para agricultor. $O$ tecnologias agroecológicas enfatizar na diversidade, sinergia, a reciclagem e integração e processos sociais que apreciam a participação da comunidade, que é o recurso humano fundamental, como a pedra angular de qualquer estratégia destinada a aumentar das opções a participação da população rural, especialmente agricultores, com poucos recursos. A abordagem agricultor a 
agricultor é precisamente, um aspecto de relevância para considerar a proposta do pensamento agroecológico, em resposta naturalmente, à inserção de profissionais técnicos como facilitadores do processo, que, em princípio, devem compreender a complexidade do sistema agrícola e as suas relações antropológicas, social, econômico, político e dos ecossistemas, a apoiar activamente os processos de geração e transferência de conhecimento. Em conclusão, os modelos agroecológicos são únicas no contexto local e tem suas peculiaridades de cada dimensão da sustentabilidade, incluindo os aspectos considerados: Cultural, ecológica, política, produtiva e econômica, entre outros; de esta maneira, definido que modelos agroecológicos são construídos a partir da análise do contexto e necessidades específicas existentes pelos produtores (sociocultural) que estão diretos responsáveis dos processos agrícolas em cada região.

Palavras-chave: Agrossistemas, pesquisa participativa, processos agroecológicas.

\section{INTRODUCCIÓN}

La mayoría de estudios relacionados con procesos agropecuarios se han direccionado a establecer las barreras biológicas y tecnológicas que limitan su desarrollo, sin considerar las relaciones entre el productor y la sociedad agraria que se supone están tratando de mejorar. La propia "neutralidad" de la ciencia proclamada por la academia, inculca en sus investigadores, la objetividad y la imparcialidad, aislándolo de la realidad social sin tener en cuenta el enfoque agroecológico que ofrece una nueva perspectiva. Los productores y su familia interactúan con los sistemas agropecuarios buscando diferentes estilos de agricultura en su interacción con la naturaleza, entre ellos y el mercado. Por lo tanto se trata no solo de experimentar con sistemas sostenibles, sino también generar cambios en los sistemas agrarios especialmente en las relaciones de producción (hombre-naturaleza; hombre-hombre), combinando los métodos de investigación cuantitativos propios de las ciencias naturales con los cualitativos de las ciencias sociales ya que en los sistemas nos encontramos unidos naturaleza y sociedad (Herrera, 2006). 
La práctica empírica forma parte importante del enfoque agroecológico, ya que asume en la diversidad de los sistemas una gran cantidad de los conocimientos necesarios para su transformación, donde se reconoce el carácter investigador de los campesinos y por tanto lo incorpora en cada fase experimental. La agroecología busca progreso endógeno de la sociedad donde se trabaja, aplicando dentro de su arsenal metodológico, la investigación- acción- participativa (IAP), (Guzmán et al., 2001). La perspectiva de la investigación agroecológica se adapta a las categorías para caracterizarla en las ciencias sociales que se estructura en tres niveles: Distributivo, estructural y dialéctica. Abordar el manejo de los recursos naturales desde una perspectiva ecológica, socioeconómica y política, no es excluyente, sino al contrario, pueden constituir niveles acumulativos que permiten una indagación cada vez más profunda de la realidad.

En primer lugar, la categoría distributiva es la forma de indagación del sistema para la obtención de datos empíricos de carácter físico, ecológicos, tecnológicos, económicos o sociales para expresarlos cuantitativamente con el mayor apoyo estadístico posible. En segundo lugar, la estructural trata de explicar las relaciones existentes entre los fenómenos analizados y los discursos desarrollados por los actores involucrados en los sistemas agropecuarios, también se genera una información cualitativa que da sentido sociocultural a los procesos generados en la realidad. En tercer lugar, la dialéctica es la articulación que se establece entre el investigador y la parte investigada, en forma crítica, en el transcurso de la transformación, lo cual supone una clara ruptura con el proceder científico convencional. Otra característica de la agroecología es el enfoque holístico y el análisis de sistema aplicado a los estudios agrarios, en la que se aplica la Teoría General de Sistemas, conocida como Farm System Research (FSR), con la cual se han diseñado modelos para el manejo de fincas y la aplicación de programas de desarrollo comunitario, y su meta es elevar la productividad del sistema agrario en un contexto de intereses sociales y privados, identificando la unidad productiva como familiar y analizando las relaciones existentes entre el medio ambiente con el humano (Collinson, 2000). 
Para realizar una adecuada investigación de los sistemas agroecológicos se deben tener en cuenta cuatro fases: 1) Diagnóstico de las explotaciones, se busca toda la información secundaria disponible, en la toma de datos de campo se emplea principalmente información suministrada por la comunidad y otras técnicas distributivas como sondeos y encuestas. 2) El Diseño que se realiza de acuerdo al diagnóstico, estableciendo los problemas a resolver con sus prioridades de investigación. 3) Evaluaciones de los resultados comparando experiencias de campo con los campesinos. 4) Proyección social una vez obtenidas las soluciones, y estando de acuerdo las tres partes implicadas (productor, investigador y extensionista), se pueden introducir estos resultados a los sistemas agroecológicos, que pueden ser utilizados en zonas con problemas similares. (Sarandón y Flores, 2014).

Se considera que el conocimiento de los sistemas agroecológicos es un proceso social, donde la participación y educación son elementos centrales para el desarrollo y la trasformación de las comunidades sobre los cuales se trabaja, que unido a la investigación dio como resultado el surgimiento de la InvestigaciónAcción-Participativa, la cual surge en los años 60, con el objetivo de procurar, a partir del conocimiento adquirido por los grupos sociales y los investigadores, y mediante su interacción, realizar las transformaciones sociales. La agroecología desarrolla un enfoque de sistema integrador, donde los componentes ecológicos, (recursos naturales), tecnológicos-económicos y los sociales son considerados a un mismo nivel (Gliessman, 2002).

\section{FUNDAMENTOS PARA LA FORMACIÓN DE UN AGROECOSISTEMA}

En cualquier sistema existen gradientes de calor o energía, y se dice que el sistema está en un equilibrio termodinámico cundo la producción y consumo es igual (Haden, 2002). Sin embargo, la realidad marca algún estado de desequilibrio termodinámico, en cualquier sistema la importación de energía a través de sus límites está dada por la exportación de entropía. La energía disponible en un sistema que condicione procesos de trabajo es una función del gradiente que existe entre el sistema y su medioambiente y su medición refleja el estado lejos 
del equilibro termodinámico. Dado que en los sistemas agropecuarios se utilizan muchas fuentes de energía almacenada o concentrada que fluye a través del ecosistema natural como resultado de un complejo conjunto de interacciones tróficas, con ciertas cantidades disipadas en diferentes puntos y momentos de la cadena alimenticia, finalmente mucha de esta energía libre, se mueve por la ruta de los desechos.

La agroecología orienta la conversión de sistemas convencionales de producción (producción intensiva, monocultivos dependientes de insumos y agroquímicos) a sistemas más diversificados y autosuficientes (Figura 1). Para esto, la agroecología utiliza principios ecológicos que favorecen procesos naturales e interacciones biológicas que optimizan sinergias de modo tal que, la agrobiodiversidad sea capaz de subsidiar por si misma procesos claves tales como la acumulación de materia orgánica, fertilidad del suelo, mecanismos de regulación biótica de plagas y la productividad de los cultivos (Gliessman, 2002). Estos procesos son cruciales pues condicionan la sustentabilidad de los agroecosistemas; la mayoría de estos procesos se optimizan mediante interacciones que emergen de combinaciones específicas espaciales y temporales de cultivos, animales y árboles, complementados por manejos orgánicos del suelo.

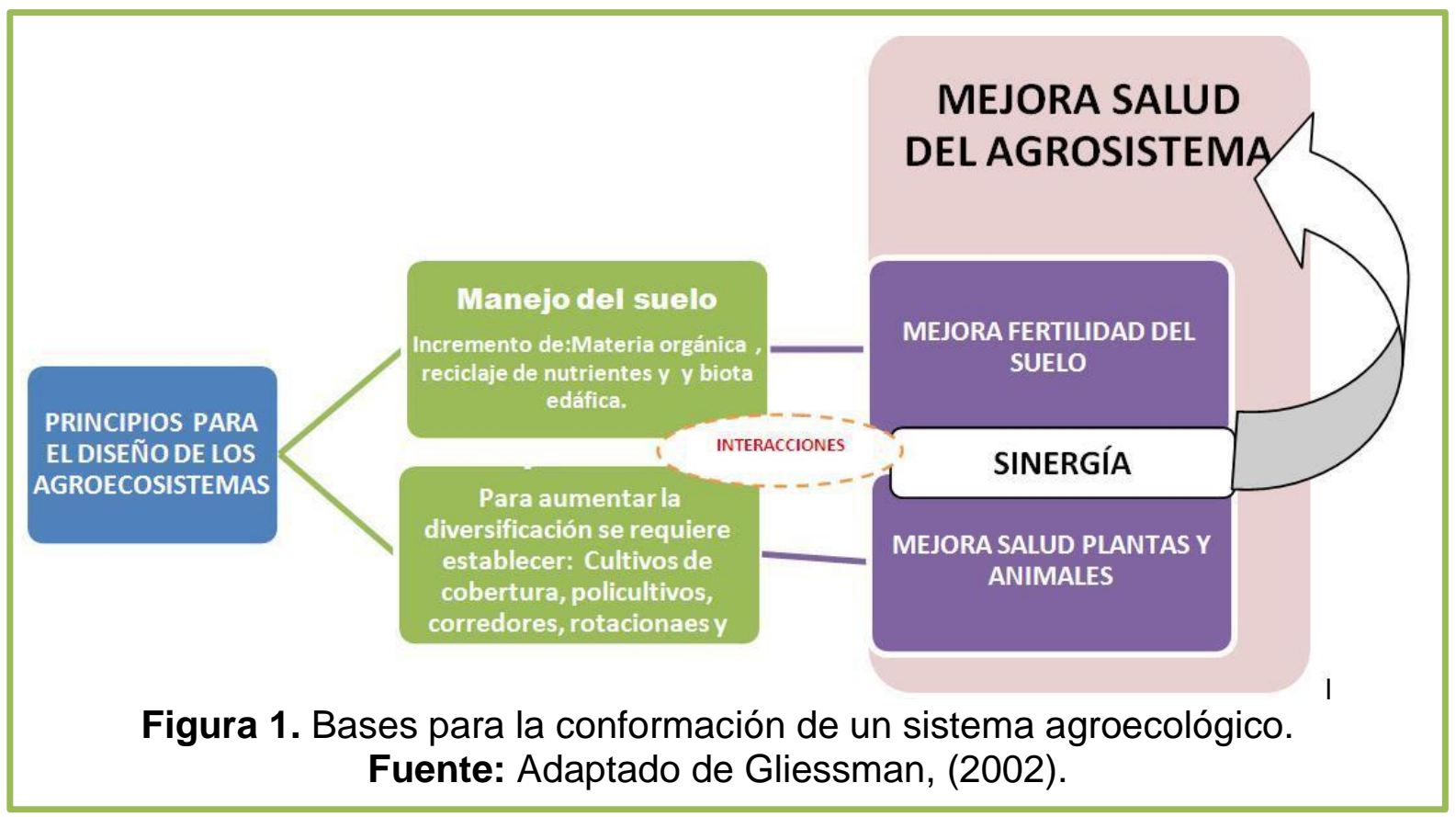




\section{PLANIFICACIÓN Y EVALUACIÓN DE UN AGROECOSISTEMA}

El punto de partida debe ser con la comunidad que va participar en esta actividad conformado un equipo de trabajo para lo cual se requiere un diagnóstico de la situación local para tener claro aspectos sociales, ambientales y económicos que determinan el éxito de una propuesta de un diseño agroecológico o de agricultura sostenible (Primavesi, 2008). Una vez organizada la comunidad, se identifican los aspectos más críticos entre los problemas para el logro de la sostenibilidad agropecuaria para las condiciones locales, para lo cual se deben realizar preguntas como: ¿Qué tan limitantes son los problemas enunciados en lo ambiental, social y económico para el conjunto de las fincas involucradas en el programa?; ¿cuáles problemas son los más relevantes para la sostenibilidad, es decir, aquellos problemas determinantes para el cumplimiento del objetivo ambiental, social o productivo, y cuáles representan los más altos riesgos para el logro de los objetivos? Hecho este análisis se seleccionan, participativamente, alrededor de cinco aspectos críticos para la sostenibilidad por cada dimensión, los principales aspectos que se consideran, por consenso, como los más riesgosos para alcanzar la sostenibilidad de los sistemas productivos (Acevedo, 2009; Primavesi, 2008) (Tabla 1).

Para conocer las variaciones es este aspecto crítico, algunas características esenciales que deben ser tenidas en cuenta al momento de formular indicadores para un sistema específico son: 1) Deben ser medibles y tangibles, 2) Recolección de información fácil y no costosa, 3) Adecuarlos al nivel de agregación del sistema, 4) Involucrar población local, 5) Centrarse en aspectos prácticos, 6) Se podrán repetir a través del tiempo, 7) Ser significativos para la sostenibilidad, 8) Ser sensibles a los cambios del sistema, 9) Analizar relaciones con otros indicadores y 10) Permitir su aplicación en otros agroecosistemas para comparar. Cada indicador debe incluir: Nombre, concepto o definición y escala de medición, (Acevedo, 2009; Primavesi, 2008). 
Tabla 1. Determinación y priorización de los puntos críticos, de un agroecosistema en Colombia

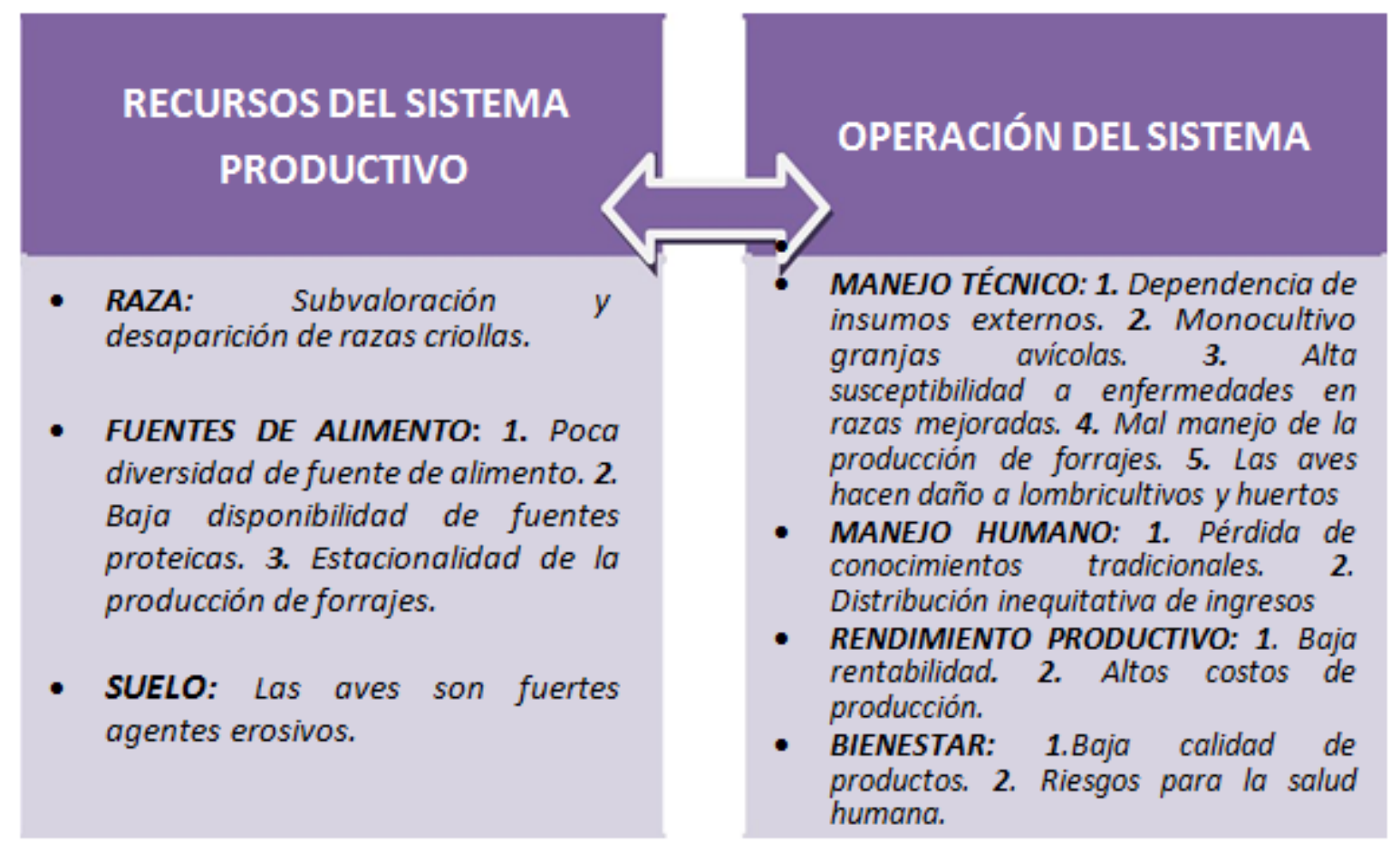

Fuente: Adaptado de Acevedo, (2009); Primavesi, (2008).

El nombre y concepto deben ser concretos y claros, de modo que al leerlo se entienda, qué es lo que pretende evaluar y cuál es su alcance; la escala debe serla sencilla de cero a cinco, siendo cero el nivel menos deseable para la sostenibilidad o el punto crítico extremo y cinco el nivel óptimo o deseable. Desde cero, y de manera gradual, se irán formulando niveles intermedios que signifiquen los pequeños pasos que se deberían ir logrando hasta alcanzar el nivel óptimo formulado. La escala también puede construirse en porcentaje dependiendo del tipo de indicadores, en tal caso el nivel máximo será 100 y puede establecerse hasta 10 niveles. Se debe comparar la sostenibilidad de una finca en varios momentos, al inicio del programa y cada vez que se aplique posteriormente la evaluación; así mismo confrontar unas fincas con otras dentro del mismo programa con predios de regiones diferentes donde se apliquen el mismo conjunto de indicadores. En cada finca se puede obtener el índice de sostenibilidad como el 
promedio de los valores obtenidos en los indicadores y se debe diagramar los datos para mejorar su comprensión (Acevedo, 2009; Primavesi, 2008).

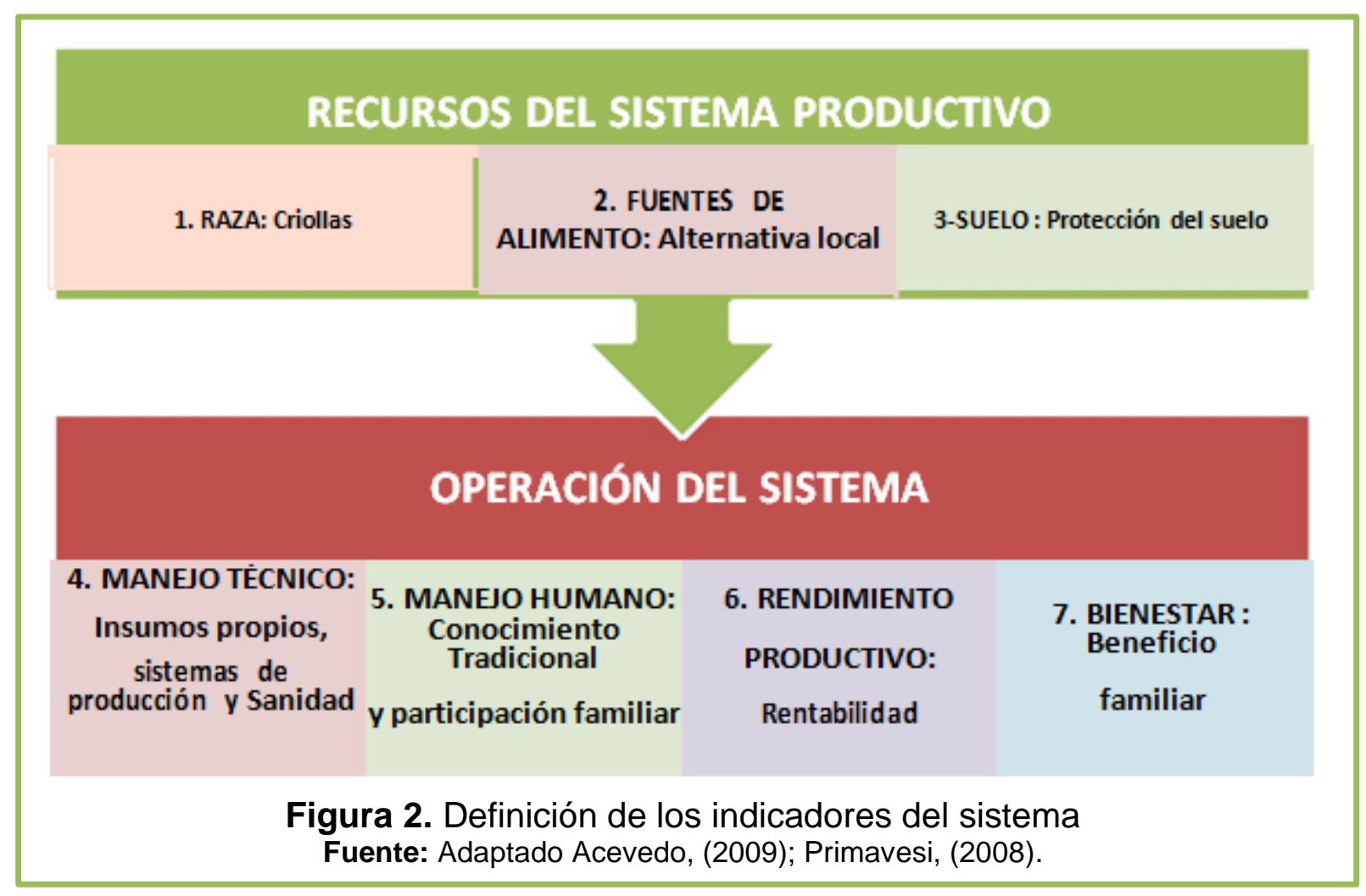

\section{ANÁLISIS CRÍTICOS DE SISTEMAS AGROECOLÓGICOS}

Indudablemente los sistemas agroecológicos están ligados a las actividades agropecuarias es un ecosistema dentro del cual ocurren otras transformaciones como: Ciclos de nutrientes, interacción de depredador/presa, competencia, comensalía y cambios sucesionales, por lo tanto, la agroecología se centra en las relaciones ecológicas en el campo y su propósito es hacer las funciones dinámicas y eficientes en esta relación. También está implícito que por medio del conocimiento de estos procesos y relaciones los sistemas agroecológicos pueden ser administrados mejor, con menores impactos negativos en el medio ambiente y la sociedad, haciéndolos sostenibles con menor uso de insumos externos. Por lo expuesto anteriormente se empieza a considerar los predios agropecuarios como un tipo especial de ecosistema llamado agroecosistema, con el fin de formalizar el análisis del conjunto de procesos e interacciones que intervienen en un sistema agrícola o pecuario. El marco analítico de la filosofía agroecológica le debe mucho 
a la teoría de sistemas y a los intentos teóricos y prácticos para integrar los numerosos factores que afectan la producción en el campo (Cerda y Sarandon, 2011).

Los agroecosistemas no están estrictamente determinados por factores de origen biótico o ambiental, sino que los elementos socioeconómicos como: Precios del mercado o cambios en la tenencia de las tierras, pueden influir en estos sistemas tan decisivamente como una sequía, explosiones de plagas o la disminución de los nutrientes en el suelo; además el manejo de la energía y recursos materiales puede aumentar la recuperación de un ecosistema dañado, aunque su administración con fines de producción a menudo han alterado en forma dramática la estructura, la diversidad, los patrones de flujo de energía y de nutrientes, y los mecanismos de control de poblaciones bióticas (Ramos et al., 2007).

La magnitud de las diferencias entre un ecosistema natural y uno agropecuario depende de la intensidad y frecuencia de las perturbaciones naturales y humanas, siendo el resultado de la interacción entre características endógenas como: Biológicas y ambientales, y de factores exógenos como: Sociales y económicos, que finalmente generan la estructura particular del agroecosistema. Por esta razón, a menudo es necesaria una perspectiva más amplia para explicar un sistema de producción que está en observación (Chapin et al., 2011).

Un sistema agropecuario difiere de un sistema ecológico natural tanto en su estructura como en su función; los agroecosistemas son semi-domesticados y han sufrido un mínimo impacto humano, y se caracterizan por: 1) Requerir fuentes auxiliares de energía, que pueden ser humana, animal y combustible para aumentar la productividad de organismos específicos. 2) La diversidad puede ser muy reducida en comparación con la de otros ecosistemas. 3) Los animales y plantas que dominan son seleccionados artificialmente y no por selección natural y 4) Los controles del agroecosistema son, en su mayoría, externos y no internos, ya que se ejercen por medio de retroalimentación del subsistema (Ramos et al., 2007). 
Considerando la interacción que se establece entre los actores sociales (grupos indígenas, campesinos, agricultores, técnicos y otros) y la naturaleza, se ha desarrollado el concepto de agroecosistema, el cual refleja la forma de apropiación por grupos humanos de los recursos naturales, y la forma como se relacionan con ellos y el exterior de los mismos, o sea el mercado, como el conjunto de explotaciones que posee una naturaleza análoga respecto a las tecnologías utilizadas, fruto de un conocimiento local común y de similares estrategias de producción, reproducción y consumo; se toma como punto de partida la capacidad estratégica de los actores locales para desarrollar los agroecosistemas o sea, lo que se denomina Capacidad Endógena desarrollada desde el agricultor familiar hasta el latifundista. Estos saberes locales contribuyen desde cómo se debe trabajar la tierra, cómo debe organizarse el hogar, (hombre, mujer e hijos), hasta cómo relacionarse con el exterior, mercado y tecnología disponible (Guzmán et al., 2001).

Por otro lado el proceso de "modernización rural", fenómeno por el cual el modo campesino de apropiación se ve transformado o sustituido por el modo agroindustrial, es realizado por un segmento periférico de la sociedad considerados como productores rurales, quienes son los encargados de "internalizar" al organismo social diversos materiales y energías de la naturaleza; para realizar esta apropiación cada unidad de producción, establece dos tipos de intercambios materiales: Ecológicos y con el resto de la sociedad a través de diferentes circuitos y mercados e intercambios económico, se asegura que para diferenciar los modos históricamente determinados de apropiación de la naturaleza que prevalecen en el mundo contemporáneo, se deben tener cuenta los siguientes aspectos: 1) Tipo de energía utilizada durante la producción, 2) Escala de las actividades productivas, 3) Grado de autosuficiencia del agroecosistema, 4) Nivel de fuerza de trabajo, 6) Diversidad (eco-geográfica, biológica y genética), 7) Productividad ecológica o energética, 8) Eficiencia del trabajo, 9) Conocimientos empleados en la apropiación/producción y 10) Visión del mundo (natural y social) que prevalece como causa invisible u oculta de la 
racionalidad productiva. Para cada factor mencionado anteriormente se buscan indicadores a los que se les asignan valores desde cero (sistema tradicional) hasta 5 (sistema agroindustrial); este entendimiento genera una herramienta muy importante para la agroecología, para entender la gran heterogeneidad de los sistema agropecuarios, influenciados por las fuerzas endógenas y exógenas que actúan en su evolución, donde es importante la escala agroecológica, para establecer la posición que ocupa, los subsistemas que lo forman y los sistemas mayores a que pertenecen o están vinculados (Vivar, 2012).

\section{Asociaciones agropecuarias (Animales-Cultivo-Árbol)}

Los sistemas especializados requieren generalmente de un flujo exógeno de recursos, y su mantenimiento y estabilidad no dependen de su capacidad propia de sustento; en cambio, los sistemas productivos agroecológicos, que miran hacia los procesos, muestran mayor dimensión de sostenimiento, generando sinergias que permiten potenciar los factores naturales para producir alimentos de origen vegetal y animal, y de restaurar el equilibrio productivo, ecológico y económico, ante la influencia negativa de cualquier factor interno o externo. En Cuba se han experimentado una serie de combinaciones y modelos de agricultura ecológica, principalmente en fincas privadas, las cuales han tenido éxito en el tiempo y sus resultados se han venido diseminando a través de los programas de transferencia de tecnología "De campesino a campesino", "Fitomejoramiento participativo", "Iniciativa para el desarrollo local", "Programa de Agricultura Urbana", "Movimiento Agroecológico de la ANAP", "Popularización del arroz y otros" (Funes, 2007).

En los últimos años se ha hecho énfasis en la reconversión de los sistemas de producción estatales, los cuales tienen las limitaciones de dependencia de los insumos externos provenientes del estado y que poseen grandes extensiones de tierra, con una agricultura o ganadería de monocultivo principalmente, lo que ha provocado una explotación indiscriminada de los recursos naturales y causado enormes impactos ambientales sobre el suelo y pérdida de biodiversidad. En este proceso de reconversión propuesto (Figura 6) denominado "modelo de sustitución de insumos", se visualiza una disminución de las áreas agrícolas dedicadas a la 
producción para exportación, cambios profundos de las estructuras productivas, una mayor orientación hacia la autosuficiencia alimentaria y por la protección del medio ambiente (Funes, 2007; Treto et al., 2002; Wright et al., 2005).

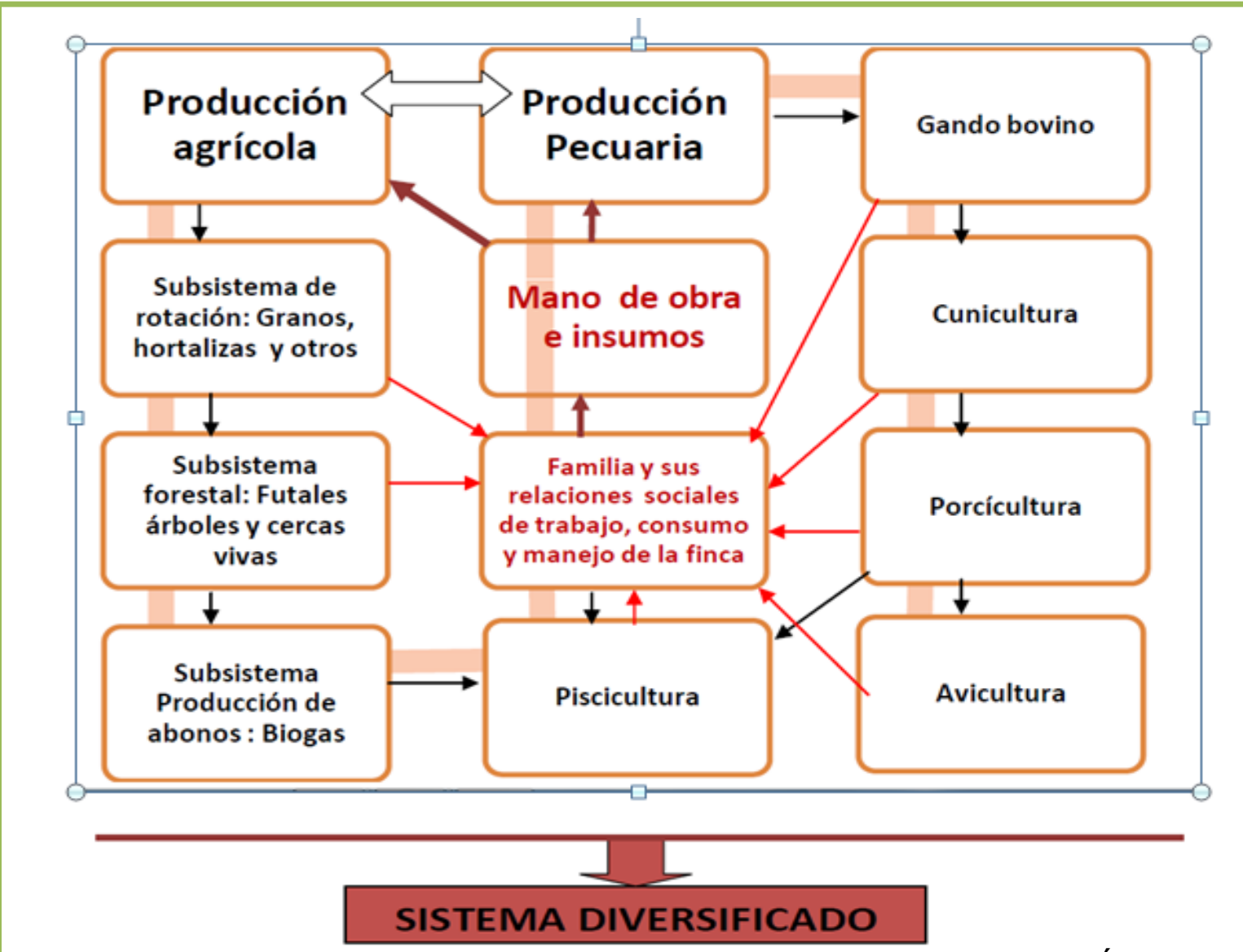

Figura 3. Modelo propuesto donde se asocian: Animales - Cultivo -Árbol Fuente: Adaptado de Funes, (2007); Treto et al., (2002); Wright et al., (2005)

Un sistema agroecológico no es considerado como la suma de sus partes, sino el resultado del todo, formado por las partes, donde se implanta no solo la racionalidad biológica del funcionamiento del suelo, las plantas y los animales, sino que se relaciona con el hombre y su entorno social, económico y político, También se desecha la visión antropocéntrica del mundo y toma lugar la visión biocéntrica, que explica el funcionamiento y evolución de cualquier cambio; otro aspecto a tener en cuenta son las técnicas y conocimientos tradicionales y los conocimientos locales, las experiencias acumuladas, incluso los conocimientos empíricos, en la actividad agropecuaria, se trasmiten de generación en generación y son de mucha importancia para lograr resultados productivos y eficientes. 
En la orientación biológica dinámica de la agroecología, los ritmos biológicos, la periodicidad entre día y noche, las fases lunares y constelaciones astrales, se aprovechan conscientemente en la producción agrícola y ganadera y sus efectos se manifiestan, evidentemente, en la calidad de los productos y en el resultado económico. En la agricultura tradicional muchas veces se enfrentan con el balance energético negativo, donde se invierte más energía que el resultado final del proceso de producción, esta energía es de fuentes no renovables como petróleo, gas natural o carbón; mientras que en la agricultura ecológica sostenible, domina el principio del uso mínimo de energía externa y del balance energético positivo (Wright et al., 2005).

\section{INVESTIGACIÓN Y EXTENSIÓN EN SISTEMAS}

La evaluación de sistemas de manejo incorporando "Indicadores de Sustentabilidad" ha sido muy empleada por agroecólogos, con el fin de analizar con índices a escala de finca desde una triple perspectiva: económica, social y ecológica, la cual sigue los siguientes pasos metodológicos: 1) Selección y caracterización previa del agroecosistema mediante la obtención de la información básica (entrevista, información secundaria) para definir el itinerario técnico, la estructura y los límites del mismo. 2) Determinación de puntos críticos que amenazan la sustentabilidad del sistema, y selección de indicadores correspondientes que están relacionados con los atributos de la sustentabilidad productividad, estabilidad, equidad, autonomía y adaptabilidad cultural. 3) Definición de los criterios operativos a considerar los máximos y mínimos admisibles. 4) Medición y monitoreo de los indicadores (ej. erosión, rendimiento, eficiencia y autonomía energética). 5) Integración de los resultados mediante análisis multicriterio y presentación en un modelo como se ilustra en la Figura 5. 6) Discusión de los resultados y recomendaciones (Sarandón y Flores, 2009).

El seguimiento de tales pasos metodológicos permite que un sistema agropecuario se adapte al paradigma agroecológico, una rápida extracción y análisis de información referente a la estructura y funcionamiento del agroecosistema, es la consideración de la sustentabilidad como propiedad emergente y la generación de 
indicadores cuantitativos y cualitativos para su evaluación de en la finca; como resultado, la información que ofrece es útil para la discusión y toma de decisiones en distintos niveles: 1) Agricultores, que pueden tomar medidas para mejorar la sustentabilidad, 2) Políticos, que tienen la posibilidad de elaborar programas que corrijan los puntos críticos que ponen en peligro la sustentabilidad del sistema, y 3) Investigadores, a los que ofrecen la posibilidad de trabajar en grupos multidisciplinares y de obtener una información sistémica clave para reconducir sus actividades experimentales disciplinares hacia la resolución de los problemas que afectan a los agroecosistemas. Esta soluciones deben ser posteriormente evaluadas igualmente desde una perspectiva sistémica y participativa, con criterios de sustentabilidad (Astier, 2007).

Sin embargo, aplicando estas metodologías se sigue presentado puntos débiles que podrían ser mejorados, como es la escasa participación del productor primario y la marginación del conocimiento tradicional en el proceso completo de análisis. También se pueden encontrar otras deficiencias de difícil mejora, entre las que se halla el hecho de que el diagnóstico obtenido es rígido, incapaz de revelar el proceso evolutivo seguido hasta llegar a la situación actual; esta información es básica para poder reconducir la producción agropecuaria actual hacia mayores niveles de sustentabilidad desde la óptica de la agroecología, que concede al principio de co-evolución de los sistemas sociales y ecológicos una dimensión central en sus bases epistemológicas (Guzmán et al., 2001).

La Investigación Acción Participativa (IAP) surge de las Ciencias Sociales en los años 40 del pasado siglo, aunque en el ámbito del trabajo con la población campesina, partió de un cuestionamiento a fondo de los sistemas de extensión y capacitación utilizados para la modernización del agro, que inició Paulo Freire con su obra ¿Extensión o Comunicación? La concientización en el medio rural (1978). La IAP parte de la base de que cualquier proceso de desarrollo que se emprenda estará sesgado, si no integra las realidades, necesidades, aspiraciones y creencias, y más aún, si no integra a los beneficiarios de este proceso como protagonista del mismo. El objetivo de la IAP es generar un conocimiento liberador 
que parte del propio conocimiento popular y que explica su realidad globalmente (enfoque sistémico), con el fin de iniciar o consolidar una estrategia de cambio (procesos de transición), paralelamente a un crecimiento del poder político, destinados ambos a alcanzar transformaciones positivas para la comunidad local; y a niveles superiores en cuanto a que es capaz de conectarse con experiencias similares (redes). El hecho de partir del conocimiento popular, de los campesinos, que sitúa su participación en el centro del proceso de investigación generando una percepción sistémica del agroecosistema, garantiza la comprensión holística de la realidad por parte del investigador. Con la IAP se ha demostrado eficacia y gran experiencia en promover cambios sociales, esto es, procesos de transición, porque es capaz de trabajar a distintos niveles: Finca, sociedad local y sociedad mayor, tal como se observa en los ejemplos de las Fotografías 1, 2 y 3 . En este sentido, intenta no sólo promover el cambio tecnológico, sino también favorecer la adquisición de capacidades y organización por parte de los grupos implicados para que puedan continuar el proceso por sí mismos, así como incidir en ámbitos superiores sociedad local o mayor (Martínez et al., 2003).
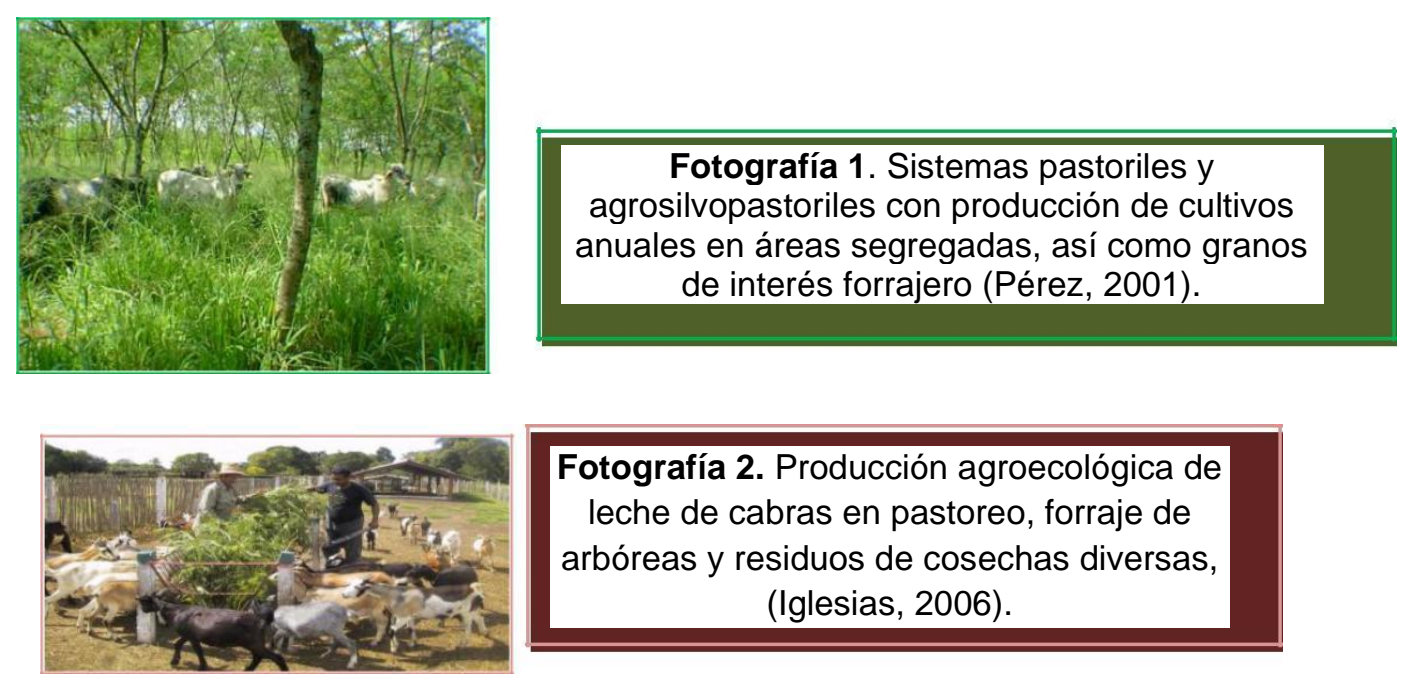

Fotografía 2. Producción agroecológica de leche de cabras en pastoreo, forraje de arbóreas y residuos de cosechas diversas, (Iglesias, 2006).
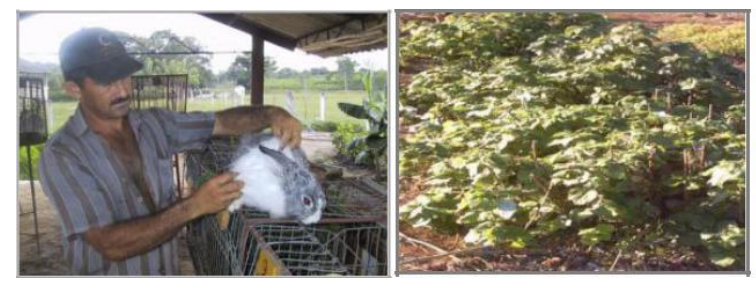

Fotografía 3. Producción cunícula y porcina con forrajeras proteicas, (Iglesias, 2006). 


\section{RELACIONES CON EL MEDIO AMBIENTE}

El estudio de la agricultura y la ganadería ha sido siempre de particular interés para la humanidad, desde hace 10.000 años las comunidades establecieron los primeros cultivos e iniciaron asentamientos permanentes, hasta el siglo XXI, en el cual la globalización es cada vez mayor, y en el que el entendimiento del funcionamiento de los sistemas agropecuarios ha sido un objetivo prioritario para nuestras sociedades; sin embargo, el interés para alcanzar dicho objetivo ha confluido en diferentes enfoques para entender los procesos que explican la estructura, funcionamiento, y actualmente, la sostenibilidad de los sistemas de productivos. El concepto clave, que guía el razonamiento metodológico y epistemológico en este análisis, es el de la sostenibilidad, definiéndola como un enfoque integral y holístico hacia la producción de alimentos, fibras y forrajes que equilibra el bienestar ambiental, la equidad social, y la viabilidad económica entre todos los sectores de la sociedad, incluyendo la comunidad internacional y a través de las generaciones. Inherente a esta definición, está la idea de que tiene que extenderse no sólo globalmente, sino también por tiempo indefinido (Gliessman, 2002).

Luego de realizar el análisis del contexto "finca", se evalúa la estructura de biodiversidad con el fin de insertar procesos que fortalezcan la inmunidad del sistema mediante las estructuras ecológicas, definiendo la biodiversidad como el conjunto de estructuras ecológicas interrelacionadas en espacios y corredores que sostienen los procesos ecológicos esenciales a través de un territorio, en sus diferentes formas e intensidades de ocupación, dotando al mismo de servicios ambientales para su desarrollo sostenible. Los tipos de biodiversidad en los sistemas agropecuarios desde la visión agroecológica son:

1) Funcional: Está asociada con el beneficio que prestan las estructuras de especies arbóreas y arbustivas, y la incorporación de materia orgánica como fuente principal del equilibrio de organismos vivos en la conservación del suelo, y al control natural de plagas y enfermedades. Varios estudios sugieren que las estructuras vegetales asociadas a campos particulares de cultivo influyen en la 
clase, abundancia y tiempo de llegada de fitófagos y sus enemigos naturales. La diversidad del mosaico vegetal de la finca, definido por la variedad de plantas cultivadas y silvestres y su estructura espacial (ej. el tamaño de los cultivos y la heterogeneidad de su distribución espacial) es un factor clave en la abundancia, diversidad y dispersión de las especies de insectos en el agroecosistema, factor que proporciona inmunidad a los cultivos (Odum, 1996).

2) Productiva: Se considera que las especies arbóreas y vegetales prestan servicios ambientales en términos de lo productivo - económico, del control natural de plagas y enfermedades, y de eficiencia energética en el sistema productivo. Un aspecto importante, es que al definir la producción a pequeña escala, se otorga mayor valor al reducir los riesgos que al elevar la producción al máximo; por lo general, los pequeños productores están más interesados en optimizar la eficiencia de los recursos o factores que les son escasos, que al incremento en la eficiencia del suelo o del trabajo, también prefieren elegir tecnologías de producción sobre la base de decisiones que toman en cuenta la totalidad del sistema agropecuario y no un cultivo en particular. El rendimiento por área puede ser un indicador, pero la productividad también puede ser medida por unidad de labor o trabajo, por unidad de inversión de dinero, en relación con necesidades o en una forma de coeficientes energéticos; cuando los patrones de producción son analizados mediante estos coeficientes, queda de manifiesto que los sistemas biodiversos son más eficientes que los agroecosistemas simples o de monocultivo en cuanto al uso de energía (Altieri y Nicholls, 2010).

3) Auxiliar: Son estructuras ecológicas adicionales, que permiten una barrera de beneficios en el sistema, para el control natural de plagas y enfermedades (ej. principios de alelopatía), aumento de controladores y aumento de la riqueza nutritiva del suelo (leguminosas rastreras), para favorecer los recursos naturales en la conformación de un agroecosistema se deben tener en cuenta:

a) Flujos de energía: Que son en forma unidireccional o irreversible entre sus componentes vivos a partir de la energía solar que entra al mismo como fuente única, su transferencia de un punto a otro se realiza con pérdidas, por 
lo tanto, no existe una eficiencia del $100 \%$ en las transformaciones de energía; en efecto, la mayor parte de la de la solar se dispersa en forma de calor; sólo cerca de un $10 \%$ de la energía de que dispone un nivel trófico es transferida próximo nivel.

b) Alta diversidad de especies: Un gran número de especies de microorganismos, vegetales y animales, que ocupan cada espacio disponible en el agroecosistema estableciendo relaciones de competencia, depredación, cooperación, comensalismo, simbiosis y otros.

c) Cadenas y redes tróficas complejas: Hay relaciones alimentarias múltiples entre todos los organismos, es así que los primarios autótrofos (vegetales) sirven de alimento a los heterótrofos y entre éstos existen diferentes niveles de interdependencia alimentaria; finalmente muchos microorganismos obtienen su energía a partir de otros en estado de descomposición. Mientras más grande es la diversidad entre los organismos autótrofos, mayor lo es entre los heterótrofos y descomponedores.

d) Arreglo espacial: Cada pequeño espacio es ocupado por especies diferentes interactuando entre sí; la distribución horizontal y vertical define una gran densidad poblacional por metro cuadrado y un aprovechamiento extraordinario del espacio superficial y aéreo.

e) Los nutrientes se mueven cíclicamente: provienen del suelo (de la transformación de las rocas) y son tomados por organismos autótrofos y, posteriormente, por los heterótrofos que al entrar al proceso de descomposición, los liberan para retornar nuevamente al suelo de donde serán extraídos nuevamente por otros organismos en proceso de desarrollo. El agroecosistema, especialmente el suelo, ha desarrollado mecanismos de retención de nutrientes dentro de sus límites físicos de modo que puedan circular sin disminuir en su cantidad.

f) Tienen un alto grado de estabilidad funcional a partir del cambio dinámico: El cambio se refiere a la gran cantidad de sucesos que ocurren dentro del ecosistema como la cantidad de energía que fluye, nutrientes que circulan de organismos que nacen o se descomponen, que se consumen entre 
sí o logran mejores niveles de adaptación; todas estas modificaciones determinan su estabilidad. Con el tiempo, la diversidad de especies, la estructura vegetativa y trófica de una comunidad, genera un equilibrio; dicha propiedad es la que permite a los ecosistemas recuperarse cuando se presentan fenómenos que distorsionan su normal funcionamiento (Altieri y Nicholls, 2010; Hiba et al., 2005).

Muchos estudios han revelado que los sistemas complejos como los de multiespecies son más fiables y sostenibles en cuanto a la conservación de recursos que los agroecosistemas simplificados. Se han reportado aumentos significativos de rendimiento en sistemas de cultivos diversos, en comparación con los monocultivos. Los rendimientos mejorados en los sistemas de cultivos y especies animales diversos (Figura 4) pueden ser resultado de una variedad de mecanismos, como un uso eficaz de los recursos (luz, agua, nutrientes), también se reduce el daño de cultivos por plagas (Altieri y Nicholls, 2010).

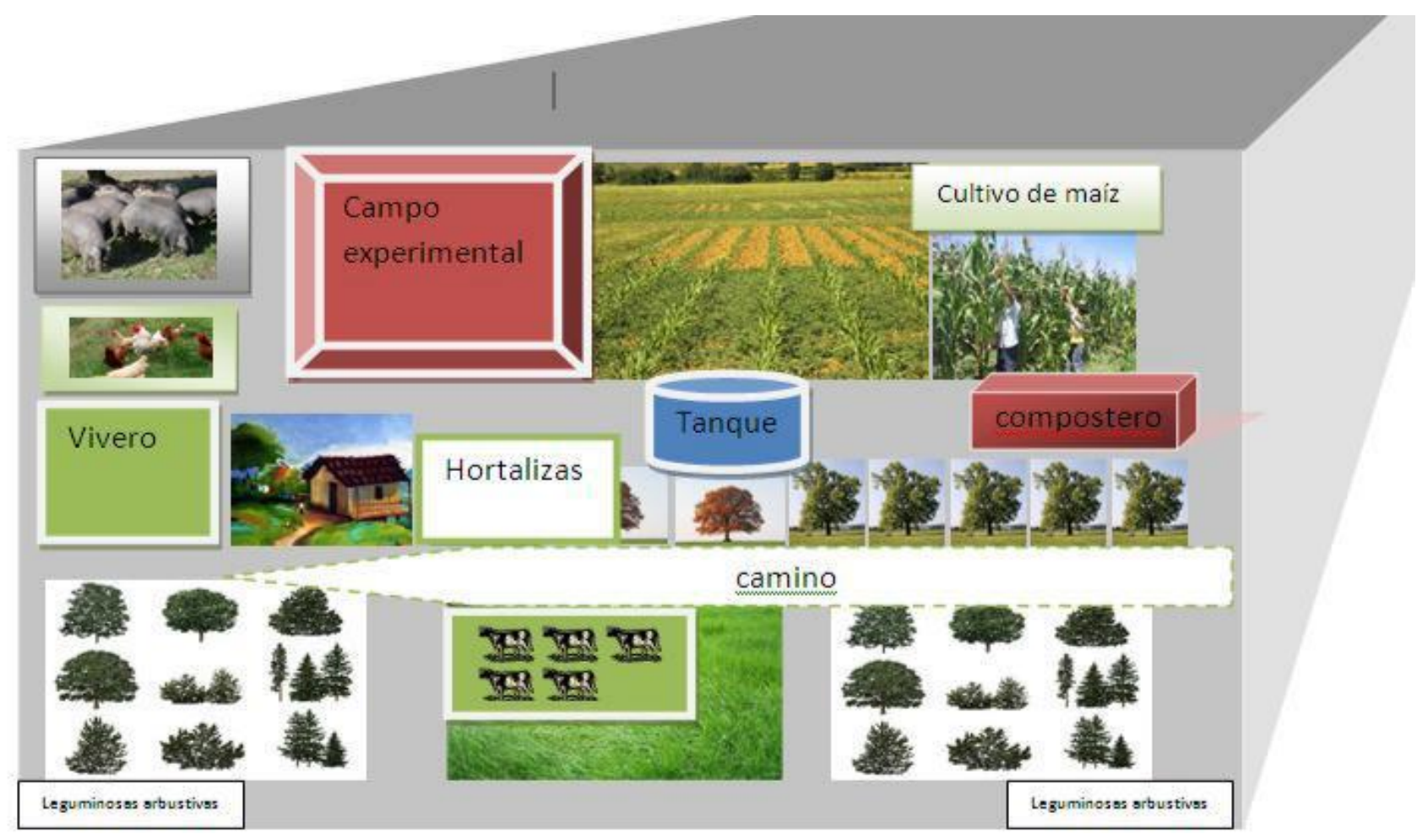

Figura 4. Formación de una finca agroecológica multiespecie a partir de un sistema tradicional.

Fuente: Adaptado de González, (2004); Hiba et al., (2005). 


\section{OPINIÓN PERSONAL DEL TEMA}

Antes de pensar en la adopción de algunos de los diseños propuestos es necesario realizar un estudio cuantitativo financiero en el cual se pueda realizar un cálculo de costos de implementación, operación y sostenimiento del sistema, para que, con base en las condiciones sociales y culturales de los productores, como acceso a créditos, disponibilidad de recursos propios, condiciones sociales y de empleabilidad en la zona, se pueda definir el riesgo y la potencialidad de asumir dicho costo.

Un elemento básico necesario para garantizar la implementación de los diseños es tener unas condiciones mínimas de asociatividad en los productores, para que lideren la búsqueda de mercados, la creación de redes productivas y comerciales, la exploración de fuentes de financiación y apoyo a productores. Desde esta perspectiva, los niveles organizacionales de las comunidades son un factor decisivo que afecta de manera directa la implementación de los diseños, pues sin organización social de base, no existe garantía que los agroecosistemas sean exitosos, y generen confianza y fidelidad en los productores.

La investigación científica sobre la diversificación de sistemas de cultivos descubre la importancia de la diversidad en un entorno de los agroecosistemas por varias razones: 1) A medida que aumenta la diversidad, también lo hacen las oportunidades para la interacción benéfica entre las especies, que pueden mejorar la sostenibilidad del agroecosistema. 2) Una mayor diversidad siempre permite un mejor uso de los recursos porque permite aumentar la heterogeneidad del hábitat, llevando a una complementariedad en las necesidades de las especies de cultivo y animales conllevando a la partición de los recursos. 3) Los ecosistemas en los cuales especies de plantas y animales están entremezclados, poseen una resistencia asociada puesto que existe una mayor abundancia y diversidad de enemigos naturales de las plagas, manteniendo bajo control las poblaciones de especies individuales de herbívoros. 4) Un ensamblaje de cultivos diversos puede crear una diversidad de microclimas dentro de los sistemas de cultivo, que pueden ser ocupados por un rango de organismos silvestres incluyendo predadores 
benéficos, parasitoides, polinizadores, fauna del suelo y antagonistas, que resultan importantes para la totalidad del sistema. 5) La diversidad reduce el riesgo para los productores, especialmente en áreas marginales con condiciones ambientales poco predecibles, puesto que sí un cultivo no anda bien, el ingreso derivado de otros puede compensarlo.

Consideró que los modelos diseñados agroecológicamente representan ventajas tanto ecológicas, como socioeconómicas, requieren atención (manejo de cultivo, podas, fertilización y otros) y manejo de la calidad del producto. Cualquier actividad productiva pensada para el mercado ya es un agro-negocio que modifica el sistema natural de la finca y por lo tanto, si el productor decide hacerlo debe responder con rigor en los temas centrales de calidad, equidad y equilibrio del ecosistema intervenido. El éxito en un agro-negocio está en la minimización del gasto energético, es decir dependencias de factores externos, tales como: insumos de síntesis química, asistencia técnica, comercialización, entre otros.

\section{CONCLUSIÓN}

A partir del análisis exploratorio a través de indicadores de sostenibilidad, se puede aumentar el nivel de ingresos familiares e incrementar la autosuficiencia alimentaria mejorando la eficiencia del subsistema agropecuario mediante la disminución de los costos, aumentando el ciclaje de nutrientes, aumentando los alimentos y superficie cultivada en el subsistema agrícola y forestal, y de esta manera se puede disminuir la dependencia de insumos generando un banco de semillas. También es importante trabajar en el diseño y la ejecución de talleres comunales en los que puedan participar habitantes de la localidad y que tengan como objetivo fundamental el fortalecimiento de redes sociales, intercambio de conocimientos, inserción en las cadenas comerciales, formación de centros comunitarios para la formación en técnicas agroecológicas. Para ello podría ser necesario evaluar aspectos como el reconocimiento de sistema de valores y creencias relacionadas a prácticas agropecuarias en su entorno, ej.: Cómo percibe la comunidad y el subsistema familia las posibles relaciones entre la agricultura y 
la alimentación, la conservación, el trabajo, la dignificación de la comunidad, el cooperativismo, entre otros.

\section{REFERENCIAS BIBLIOGRÁFICAS}

1. Acevedo Á. ¿Cómo evaluar el nivel de sostenibilidad de un programa agroecológico? Un procedimiento metodológico para diseñar, monitorear y evaluar programas rurales con enfoque de desarrollo sostenible. Bogotá, Colombia, Universidad de la Amazonia, 2009. Disponible En: http://www.udla.edu.co/documentos/docs/Programas\%20Academicos/Ingenieri a\%20Agroecologica/Memorias/1\%20Simposio\%20Internacional\%20de\%20Agr oecologia/Construccion\%20Indicadores\%20Sostenibilidad.\%20A.Acevedo.pdf

2. Altieri M.A., Nicholls C. Diseños agroecológicos para incrementar la biodiversidad de entomofauna benéfica en agroecosistemas. Sociedad Científica Latinoamericana de Agroecología. 1: 82. 2010.

3. Astier M., Curso internacional de Agroecología, Evento efectuado en la Facultad de Agricultura de la Universidad de Antioquia., Medellín, Colombia, 2007.

4. Cerda E., Sarandon S.J. Aplicación del enfoque de la Agroecología para el manejo sustentable de sistemas extensivos de clima templado. El caso de "La Aurora" en el sudeste de la provincia de Buenos Aires, Benito Juárez. Argentina. Cuadernos de Agroecología. 6 (2): 6 p. 2011.

5. Collinson M.P. A history of farming systems research. Food and Agriculture Organization of the United Nations and CABI Publishing, United Kingdoom. 2000.

6. Chapin I., F Stuart, Matson P.A., Vitousek P. Principles of terrestrial ecosystem ecology. Springer Science \& Business Media, New York, USA. 528 p. 2011.

7. Funes F. Towards sustainable agriculture in Cuba. Universidad de Salamanca, $24 \quad$ p. $2007 . \quad$ Disponibles En: http://campus.usal.es/ ehe/Papers/Microsoft\%20Word\%20-

\%20Towards\%20sustainable\%20agriculture\%20in\%20Cuba\%201st\%20Augus t\%5b1\%5d.pdf

8. Gliessman S.R. Agroecología: procesos ecológicos en agricultura sostenible. CATIE, Costa Rica. 361 p. 2002.

9. González L. La extensión agraria en Cuba. Algunas reflexiones necesarias. Pastos y forrajes. 27 (3): 211-218. 2004.

10. Guzmán C.G., González d.M.M., Sevilla G.E. Introducción a la agroecología como desarrollo rural sostenible. Revista Española de Investigaciones Sociológicas. 95: 213-217. 2001.

11. Haden A.C. Emergy analysis of food production at S\&S homestead farm. S\&S Center for Sustainable Agriculture, Lopez Island, WA, USA. p 1-33. 2002.

12. Herrera F.T. Innovaciones tecnológicas en la agricultura empresarial mexicana. Una aproximación teórica. Gaceta Laboral. 12 (1): 2006.

13. Hiba J.C., Ciciliani A., Cóppola A. Cómo mejorar las condiciones de trabajo y la productividad en empresas agrícolas y agroindustriales: Guía para la acción. Organización Internacional del Trabajo, Buenos Aires, Argentina. 197 p. 2005. 
14. Martínez A.C., Mendoza H.M.T., Alvarez M.M.R. Análisis multivariado: un manual para investigadores. Editorial Biblioteca Nueva, Barcelona, España. 120 p. 2003.

15. Odum H.T. Environmental accounting: EMERGY and environmental decision making. Jhon Wiley \& Sons, Inc, New York, USA. 372 p. 1996.

16. Primavesi A.M. Agroecologia e manejo do solo. Revista Agriculturas: Experiências em Agroecología. 5 (3): 7-10. 2008.

17. Ramos A.E., Ojeda R., Porto F., León O., Romero I., Estéves Y., Oramas J., Pérez A., Martínez Y., Reiner M. Actividad física, agroecología y calidad de vida. Revista Digital - Buenos Aires. 12 (109). 2007.

18. Sarandón S.J., Flores C.C. Evaluación de la sustentabilidad en agroecosistemas: una propuesta metodológica. Agroecología. 4 19-28. 2009.

19. Sarandón S.J., Flores C.C. Agroecología bases teóricas para el diseño y manejo de agroecosistemas sustentables. 467 p. 2014.

20. Treto E., García M., Viera R.M., Febles J.M. Advances in organic soil management. Sustainable agriculture and resistance: Transforming food production in Cuba, Food First, Oakland. p 164-189. 2002.

21. Vivar J. Feminización y valorización del trabajo. Un análisis desde las prácticas laborales y el uso de las herramientas. Textual. (60): 61-80. 2012.

22. Wright P., Triggs C., Burge G. Control of bacterial soft rot of calla (Zantedeschia spp.) by pathogen exclusion, elimination and removal. New Zealand Journal of Crop and Horticultural Science. 33 (2): 117-123. 2005. 